\title{
Línea de base para evaluar el impacto de una planta de celulosa en el Río Uruguay
}

Saizar, C. ${ }^{(1)}$, Míguez, D. ${ }^{(2)}$, Dabezies, M. ${ }^{(1)}$, Teixeira-de Mello, F. ${ }^{(3)}$, Clemente, J. ${ }^{(1)}$, Ferrari, G. ${ }^{(1)}$, Boccardi, L. ${ }^{(1)}$, Tana, J. ${ }^{(4)}$.

(1) Departamento Medio Ambiente. Laboratorio Tecnológico del Uruguay, LATU - (2) Departamento Aguas y Productos Químicos. Laboratorio Tecnológico del Uruguay, LATU - ${ }^{(3)}$ Grupo de Ecología y Rehabilitación de Sistemas Acuáticos. CURE - Facultad de Ciencias, Universidad de la República, Uruguay - ${ }^{(4)}$ AF - Consult Ltd, Rajatorpantie 8, Fl-01600 Vantaa, Finlandia.

Contacto: csaizar@latu.org.uy

Recibido: 15/07/2010 - Aprobado: 26/11/2010

\begin{abstract}
$\underline{\text { Resumen }}$
Como parte del proceso de evaluación del impacto ambiental ante la puesta en funcionamiento de la planta de pulpa de celulosa Kraft Botnia (actual UPM), se realizaron estudios de línea de base entre los años 2005 y 2007 . El diseño de muestreo incluyó tres transectas perpendiculares a la línea de costa, en tres sitios: Nuevo Berlín, Fray Bentos y Las Cañas. Se realizaron estudios de parámetros físico-químicos del agua (nutrientes, sustancias orgánicas, metales, compuestos orgánicos adsorbibles (AOX), dioxinas y furanos), materia orgánica y granulometría del sedimento, estudios biológicos de las comunidades planctónicas, zoobentónicas e ícticas y bioacumulación de dioxinas en peces. El fósforo en la columna de agua presentó en promedio valores por encima de lo recomendado por la normativa nacional, mientras los contaminantes orgánicos (AOX, clorofenoles, ácidos resínicos, fitosteroles, dioxinas y furanos) se mantuvieron por debajo de los valores guía recomendados a nivel internacional. Las comunidades planctónicas variaron significativamente entre muestreos, la de peces entre sitios y muestreos, mientras que la de bentos no presentó variaciones significativas espaciales ni temporales. El bagre trompudo (Iheringichthys labrosus) es propuesto como especie a ser monitoreada debido a su ubicuidad y abundancia. Este trabajo ofrece una visión general de los estudios de línea de base, destacando la multiplicidad y la periodicidad de los parámetros en dos años de estudio.

Palabras clave: Planta celulosa, calidad agua, comunidades biológicas.
\end{abstract}

\begin{abstract}
$\underline{\text { Abstract }}$
In the framework of the environmental impact assessment process undertaken for the commissioning of the Kraft Botnia (currently UPM) paper mill, baseline studies were conducted between 2005 and 2007. The sampling design included three transections perpendicular to the coast line at three sites: Nuevo Berlín, Fray Bentos and Las Cañas. The work included testing of physico-chemical parameters of water (nutrients, organic matter, metals, adsorbable organic compounds (AOX), dioxins and furanes), sediment organic matter and granulometry, biological studies of the planktonic, zoobenthonic and fish communities, and bioaccumulation of dioxins in fish. As an average, phosphorus in the water column presented values above those recommended by the national norms, while organic pollutants (AOX, chlorophenols, resinic acids, phytosterols, dioxins and furanes) remained below the guidance values recommended at an international level. Planktonic communities varied significantly across samplings; fish communities varied between sites and samplings, while the benthos results did not show significant space or time variations. The hag Iheringichthys labrosus has been proposed as the most suitable indicator species to be monitored, owing to its ubiquity and abundance. This paper offers an overview of the baseline assessments, highlighting the multiplicity and periodicity of the parameters collected in the course of two years.

Keywords: Paper mill, water quality, biological communities.
\end{abstract}

\section{Introducción}

Las herramientas más usadas para evaluar impactos en los ecosistemas acuáticos son aquellas basadas en las características físicas, químicas y biológicas (Karr, 1981; Davenport y Sayer, 1993). Las variables fisicoquímicas aportan información referente a las posibles causas de la contaminación, mientras que las comunidades biológicas brindan una visión integrada de los efectos a mediano y largo plazo (Hutson y Roberts, 1994). En las últimas décadas, ha recibido especial atención el uso de indicadores biológicos como una herramienta potencialmente poderosa en la evaluación de la salud ambiental (USEPA, 2002; Hering et al., 2004). La generación temporal y espacial de información físico-química y biológica de forma combinada proporciona una visión general de las características y el funcionamiento del ecosistema, lo cual permite desarrollar o definir descriptores para evaluar los cambios espacio-temporales del sistema.

El estudio de las condiciones ambientales antes del inicio de una actividad humana se puede definir como estudio de línea de base. Estos estudios se deben realizar a una escala temporal y espacial adecuada para registrar los procesos que regulan la dinámica de las comunidades biológicas, con el fin de compararlo con los datos de seguimiento luego que las actividades hayan comenzado. Los mismos se basan en posteriores comparaciones entre los sitios afectados y los de control, permitiendo determinar la presencia/ausencia de organismos y/o grados de impacto. Los efluentes de plantas de celulosa pueden generar ecotoxicidad y alterar el estado ecológico de un curso de agua. La materia orgánica y los nutrientes aportados por los efluentes pueden producir eutrofización, aumentar la conductividad, acumular taninos y lignina e inclusive alterar el curso de agua desde el punto de vista microbiológico (Karrash et al., 2006). Los efectos 
de toxicidad crónica incluyen efectos de disrupción endocrina que pueden ocasionar cambio del sexo de los peces (Larsson y Forlin, 2002). Algunos de los posibles compuestos responsables serían los ácidos resínicos y los esteroles provenientes de la degradación de la lignina, así como también los compuestos orgánicos clorados tales como dioxinas y furanos y clorofenoles provenientes del blanqueo de la celulosa (Ali y Sreekrishnan, 2001).

El Río Uruguay es un curso de agua internacional cuya cuenca hidrográfica forma parte de los territorios de Argentina, Brasil y Uruguay, abarcando un área de aproximadamente $339.000 \mathrm{~km}^{2}$. Tiene una longitud de $1.800 \mathrm{~km}$ y se extiende desde la Sierra do Mar (Brasil) hasta su desembocadura en el Río de la Plata. Aproximadamente el $30 \%$ corresponde al tramo binacional uruguayo-argentino, el cual se encuentra administrado por la Comisión Administradora del Río Uruguay (CARU), órgano binacional formado en 1975 a partir de la suscripción del "Estatuto del Río Uruguay" (CARU, 2009). Los estándares de calidad del agua que se deben cumplir en el Río Uruguay son los propuestos por el Digesto de la Calidad del Río Uruguay de la CARU. La normativa define estándares de calidad clasificados en cuatro clases, en base a los usos preponderantes de las aguas del río: abastecimiento público, recreación, actividades agropecuarias y conservación y desarrollo de la vida acuática. En el caso de los parámetros que no están incluidos en la normativa nacional se usan como referencia niveles guía internacionales, principalmente los valores guía de calidad de agua recomendados por organismos ambientales canadienses.

Este trabajo resume los resultados de los estudios de línea de base de calidad del agua y comunidades biológicas (plancton, bentos y peces) realizados por el LATU durante los años 2005 a 2007, en la zona donde posteriormente fue instalada una fábrica de celulosa (tramo inferior del Río Uruguay).

\section{Materiales y Métodos}

El área de estudio se ubica en el tramo inferior del Río Uruguay, que se extiende entre el embalse de Salto Grande y la Punta Gorda, en el departamento de Colonia. Las muestras fueron obtenidas en tres zonas de este tramo (Figura 1). La primera zona se encuentra a la altura de la ciudad de Nuevo Berlín, $30 \mathrm{~km}$ al norte de la planta de celulosa. La zona Fray Bentos y la bahía de Yaguareté se ubican junto a la proyectada descarga de los efluentes de la planta y Las Cañas se sitúa $15 \mathrm{~km}$ aguas abajo de la planta. En los muestreos de calidad de agua se adicionó un punto a la altura del puente internacional de Fray Bentos, $3 \mathrm{~km}$ al norte de la planta de celulosa (marcado con una línea negra en la Figura 1).

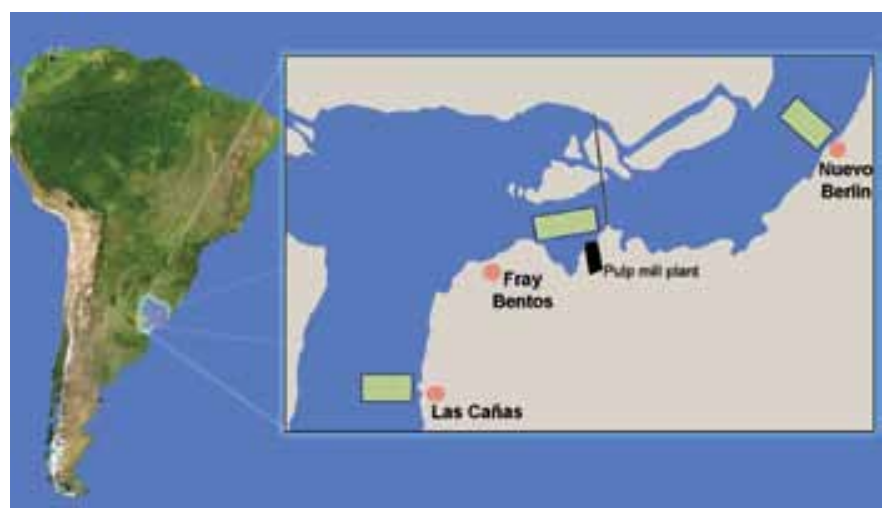

Figura 1. Ubicación de las zonas de estudio en el Río Uruguay. La línea negra marca la ubicación del puente internacional.

El período de estudio se extendió de abril de 2005 a abril de 2007 para los parámetros de calidad de agua y la comunidad de peces, y de julio de 2006 hasta abril de 2007 para la calidad de sedimento y las comunidades de plancton y bentos. La frecuencia de estudio fue mensual para los parámetros de calidad de agua y estacional para las comunidades biológicas y sedimentos (Tabla 1).

\begin{tabular}{|l|c|l|}
\hline Tipo de muestreo & Frecuencia & \multicolumn{1}{|c|}{ Meses } \\
\hline Calidad de agua & Mensual & Abril 2005 - Abril 2007 \\
\hline Plancton & Estacional & $\begin{array}{l}\text { Julio 2006, Noviembre 2006, } \\
\text { Febrero 2007, Abril 2007 }\end{array}$ \\
\hline Bentos y sedimento & Estacional & $\begin{array}{l}\text { Julio 2006, Noviembre 2006, } \\
\text { Febrero 2007, Abril 2007 }\end{array}$ \\
\hline Peces & Semestral & $\begin{array}{l}\text { Abril (2005, 2006, 2007), } \\
\text { Diciembre (2005, 2006) }\end{array}$ \\
\hline
\end{tabular}

Tabla 1. Frecuencia de los diferentes tipos de muestreo.

Las muestras para el monitoreo mensual de la calidad del agua fueron tomadas de forma directa de la capa subsuperficial del río, preservadas y analizadas en laboratorio en base a métodos estandarizados y acreditados (Tabla 2). En la Tabla 2 se presentan los parámetros analizados en el muestreo mensual de calidad de agua. Las muestras de microcistina se obtuvieron en enero y febrero de 2006 en el marco del monitoreo de calidad de agua, mediante la metodología planteada por Lawton (1994). En el marco de los estudios de la comunidad de peces se tomaron muestras de agua para analizar fenoles clorados, ácidos resínicos, fitoesteroles y dioxinas. En el sedimento se analizó la granulometría y materia orgánica por medio de las metodologías planteadas por Burt (2004) y USEPA (1995), respectivamente.

Para el estudio de las comunidades biológicas y calidad del sedimento, el diseño de muestreo constó de tres transectas perpendiculares a la línea de costa (aproximadamente $300 \mathrm{~m}$ de distancia entre ellas) en cada una de las zonas de estudio descritas. Las muestras cuantitativas de plancton fueron obtenidas en tres puntos (litoral, central y canal) de la transecta central con botella Van Dorne, realizando sucesivas extracciones en la capa eufótica hasta obtener 40 litros de agua. Se obtuvieron muestras para fitoplancton, fijadas con lugol, y el volumen restante fue filtrado por una red de $63 \mu \mathrm{m}$ de malla para analizar zooplancton. Las muestras cualitativas se colectaron mediante arrastre oblicuo subsuperficial con redes de 20 y $63 \mu \mathrm{m}$ de malla, para fitoplancton y zooplancton, respectivamente. Las muestras de zooplancton y cualitativas de fitoplancton fueron fijadas in situ con formaldehído al $4 \%$.

Las muestras de plancton fueron analizadas bajo microscopio invertido (100-1000X) con la metodología de Utermhöl (1958) en cámaras de sedimentación de 5,10 y $25 \mathrm{ml}$ (fitoplancton) y las de zooplancton en cámaras SedgwickRafter y de Bogorov. Se calculó la densidad de organismos por unidad de volumen: el fitoplancton en células/ml y el zooplancton en organismos/litro.

Las muestras para calidad de sedimento y análisis de macrozoobentos fueron obtenidas en todos los puntos de las tres transectas definidas con draga Petit Ponar. Las muestras de sedimentos fueron conservadas en frío y las biológicas fueron tamizadas en campo por una malla de $500 \mu \mathrm{m}$ de abertura y preservadas en alcohol al $70 \%$. Los organismos fueron identificados y cuantificados bajo lupa esteroscópica Arcano hasta el nivel taxonómico de familia y se calculó la densidad como individuos $/ \mathrm{m}^{2}$. Para evaluar diferencias espaciales (zonas y sitios) y temporales (entre muestreos) de las comunidades planctónicas y bentónica se realizaron análisis no paramétricos de varianza (ANOSIM) con las matrices de densidad de cada comunidad.

Los muestreos de la comunidad de peces y los estudios en bilis de peces fueron realizados en las mismas zonas que los estudios de calidad de agua, plancton y bentos (Nuevo Berlín, Fray Bentos y Las Cañas). Los resultados son expresados como captura por unidad de esfuerzo (CPUE). Los primeros tres muestreos fueron de ajuste de la metodología, sobre todo del número de redes a utilizar. En estos muestreos los peces colectados no se separaron por réplicas. Finalmente, el muestreo se estandarizó y se ha mantenido desde diciembre de 2006 de la siguiente manera: se utilizaron ocho series de redes Nórdicas estandarizadas según la norma EVS-EN 14757:2005 en cada uno de los sitios; cada una tiene $30 \mathrm{~m}$ de largo y $1.5 \mathrm{~m}$ de alto y están compuestas por 12 tramos de $2.5 \mathrm{~m}$ con diferentes tamaños 
de malla $(5.0,6.25,8.0,10,12.5,15.5,19.5,24,29,35,43$ y $55 \mathrm{~mm}$ entre nudos). En esta estandarización del muestreo se trató de cubrir un amplio rango espacial y temporal. Espacialmente, se optó por colocar en cada sitio cuatro de las redes en la zona litoral (aproximadamente 100 metros de la línea de costa) y cuatro redes en una zona más aguas abiertas (aproximadamente 300-500 metros de la línea de costa). Temporalmente, en cada época de muestreo, el procedimiento se repite exactamente igual siete días después; por lo tanto el esfuerzo de muestreo final en cada sitio es de 16 redes. Los datos que se muestran en este trabajo son la media de las CPUE, agrupando las capturas de esta secuencia espacial y temporal. Las redes fueron caladas durante 12 horas desde las 8 p.m. hasta las 8 a.m.

En función de la selección del Bagre trompudo como especie indicadora se estudiaron el índice somático, factor de condición e índice gonadosomático en las tres zonas, en abril de 2007, en hembras de las clases de tamaño 15-22 capturadas en las tres áreas de estudio. Los resultados se compararon con el test de Kruskal-Wallis para un límite de confianza del $95 \%$.

Las muestras de bilis fueron colectadas en diferentes especies, con jeringa de $1 \mathrm{ml}$ y luego depositadas en vial de $5 \mathrm{ml}$ con membrana de teflón. Se tomó una muestra compuesta para cada especie seleccionada a partir de 3-8 peces, lo cual dependió del tamaño de los individuos. Por otra parte, se tomaron muestras de músculo compuestas (3-5 peces) en diferentes especies para el análisis de dioxinas. Las muestras de músculo de aproximadamente $200 \mathrm{~g}$, que corresponden a una sección transversal en la zona media del pez, se guardaron en papel de aluminio y se congelaron.

\begin{tabular}{|c|c|c|}
\hline Parámetro & Método & LD (mg/l) \\
\hline Temperatura & Medido in situ con termocupla, basado en APHA 2550B & NA \\
\hline Conductividad & PEC.PQAR.112, basado en ISO 7888-1985-05-15 & NA \\
\hline $\mathrm{pH}$ & PEC.PQAR.101, basado en APHA 4500H+B & NA \\
\hline Color (como Pt) & ISO 788720 ed., Sección 4 & 2,5 \\
\hline Oxígeno disuelto $\left(\right.$ como $\left.\mathrm{O}_{2}\right)$ & ISO 5814:1990 & 0,2 \\
\hline Turbiedad (FNU) & ISO 7027:1999 & 0,05 \\
\hline Sólidos disueltos totales & PEC.PQAR004, basado en APHA 2540C & 6 \\
\hline Sólidos suspendidos totales $(\mathrm{mg} / \mathrm{l})$ & PEC.PQAR006, basado en APHA 2540D, equivalente a ISO 11923:1997 & 5 \\
\hline Dureza total $\left(\mathrm{como} \mathrm{CaCO}_{3}\right)$ & $\begin{array}{l}\text { PEC.PQAR106, basado en APHA 2340C, } \\
\text { equivalente a ISO 6059-1984 (E) }\end{array}$ & 0,6 \\
\hline Cloruros (como $\mathrm{Cl}$ ) & ISO 10304/1-1992 & 0,2 \\
\hline Sulfatos (como $\left.\mathrm{SO}_{4}\right)$ & ISO $10304 / 1-1992$ & 0,1 \\
\hline Nitrato (como N) & ISO 10304/1-1992 & 0,06 \\
\hline Nitrito (como N) & ISO 6777:1984 (E) & 0,002 \\
\hline Nitrógeno Kjeldahl total (como N) & ISO 5663-1984 (E) / ISO 6778:1984 & 0,5 \\
\hline Nitrógeno total (como N) & ISO 905-2, con electrodo & 0,5 \\
\hline Amonio (como N) & ISO 6778:1984 & 0,05 \\
\hline Fósforo total (como P) & ISO 6878:2004 & 0,005 \\
\hline Fósforo soluble (como P) & ISO 10304/1-1992 y APHA 4500-P-D & 0,0002 \\
\hline Sílice reactiva soluble (como $\mathrm{SiO}_{2}$ ) & APHA $4500-\mathrm{SiO}_{2} \mathrm{C}$ & 0,001 \\
\hline Cromo hexavalente (como $\mathrm{Cr}$ ) & APHA $3500 \mathrm{Cr}$ B & 0,05 \\
\hline Sulfuros disueltos (como S) & ISO 10530 & 0,007 \\
\hline Mercurio & ISO 5666 (CVACOMO) & 0,0002 \\
\hline Sodio & ISO 9964-1 (FACOMO) & 0,010 \\
\hline Potasio & ISO 9964-2 (FACOMO) & 0,020 \\
\hline Cobre & ISO 8288 (FACOMO) & 0,006 \\
\hline Zinc & ISO 8288 (FACOMO) & 0,004 \\
\hline Manganeso & $\begin{array}{c}\text { ISO } 8288 \text { (FACOMO) adapted-ISO 15587-2 } \\
\text { Annex C (Nitric acid digestion) }\end{array}$ & 0,008 \\
\hline Hierro & $\begin{array}{c}\text { ISO } 8288 \text { (FACOMO) adapted-ISO 15587-2 } \\
\text { Annex C (Nitric acid digestion) }\end{array}$ & 0,011 \\
\hline Oxidabilidad (Índice de permanganato) $\left(\right.$ como $_{2}$ ) & ISO 8467:1993 (E) & 0,5 \\
\hline Demanda Química de Oxígeno (DQO, como $\mathrm{O}_{2}$ ) & ISO 15705:2002 & 0,014 \\
\hline Carbono orgánico total (como C) & USP29, ISO 8245 & 0,15 \\
\hline Demanda Bioquímica de Oxígeno $\left(\mathrm{cDBO}_{7}\right.$, como $\left._{2}\right)$ & ISO 5815/2:2003 (E) & 0,5 \\
\hline Aceites y grasas & APHA 5520 D & 10 \\
\hline Sustancias fenólicas (como $\left.\mathrm{C}_{6} \mathrm{H}_{5} \mathrm{OH}\right)(\mu \mathrm{g} / \mathrm{l})$ & ISO 6439:1990 & 0,001 \\
\hline Compuestos orgánicos adsorbibles (AOX) & EN ISO 9562:2004 & 0,008 \\
\hline
\end{tabular}

Tabla 2. Parámetros de calidad de agua estudiados, métodos usados y límites de detección (LD) (NA: No aplica). 


\section{Resultados}

La variación de los parámetros medidos se presenta en la Tabla 3. Las concentraciones de nitrato fluctuaron entre 0.04 y $1.4 \mathrm{mg} / 1$ y las de fósforo total entre 0.01 y $0.6 \mathrm{mg} / \mathrm{l}$.

\begin{tabular}{|c|c|c|c|c|c|}
\hline Parámetro & Unidad & Nuevo Berlín & Puente & Fray bentos & Las Cañas \\
\hline Temperatura & ${ }^{\circ} \mathrm{C}$ & $21,7 / 14,7-32,5$ & $21,8 / 14,9-31,5$ & $21,8 / 14,8-30,7$ & $21,6 / 14,8-29,8$ \\
\hline Oxígeno disuelto & $\mathrm{mg} / \mathrm{l}$ & $8,5 / 6,8-9,8$ & $8,6 / 7,2-9,7$ & $8,5 / 7,4-9,7$ & $8,6 / 7,4-9,9$ \\
\hline Conductividad & $\mu \mathrm{S} / \mathrm{cm}$ & 69/49-109 & 69/48-149 & 69/54-103 & $75 / 53-153$ \\
\hline $\mathrm{pH}$ & & $7,5 / 7,0-8,3$ & $7,6 / 7,0-9,1$ & $7,7 / 7,1-9,0$ & $7,6 / 7,0-9,2$ \\
\hline Color (como Pt) & $\mathrm{mg} / \mathrm{l}$ & $73 / 30-125$ & $75 / 30-125$ & $74 / 30-125$ & $73 / 30-125$ \\
\hline Turbiedad (FNU) & FNU & $19,3 / 7,2-36,9$ & $20,5 / 9,3-59,0$ & $17,8 / 9,4-35,0$ & $21,4 / 8,5-49,0$ \\
\hline Sólidos suspendidos totales (mg/l) & $\mathrm{mg} / \mathrm{l}$ & $12,0 /<5-28,5$ & $11,0 /<5-32,5$ & $8,6 /<5-18,6$ & $14,3 /<5-60,3$ \\
\hline Sólidos disueltos totales & $\mathrm{mg} / \mathrm{l}$ & $76,7 / 16,5-145,0$ & $73,5 / 27,5-158,0$ & $75,7 / 27,5-136,0$ & $78,9 / 29,5-128,0$ \\
\hline Carbono orgánico total (como C) & $\mathrm{mg} / \mathrm{l}$ & $3,0 / 1,5-5,8$ & $3,2 / 1,5-7,5$ & $3,1 / 0,6-5,8$ & $3,4 / 0,8-15,7$ \\
\hline $\mathrm{DBO}_{7}$ & $\mathrm{mg} / \mathrm{l}$ & $1,6 /<0,5-4,8$ & $1,8 /<0,5-5,2$ & $1,8 /<0,5-5,7$ & $2,0 /<0,5-15,7$ \\
\hline Nitrógeno total (como N) & $\mathrm{mg} / 1$ & $0,67 / 0,01-2,4$ & $0,64 / 0,07-2,20$ & $0,70 / 0,04-2,00$ & $0,80 / 0,07-2,40$ \\
\hline Fósforo total $($ como $\mathrm{P}){ }^{\natural}$ & $\mu \mathrm{g} / 1$ & $68 / 26-121$ & 70/11-197 & $66 / 25-118$ & $68 / 27-103$ \\
\hline Nitrato & $\mathrm{mg} / 1$ & $0,51 / 0,12-1,04$ & $0,54 / 0,05-1,12$ & $0,53 / 0,07-1,430$ & $0,51 / 0,04-1,00$ \\
\hline Nitrito & $\mu \mathrm{g} / \mathrm{l}$ & $9,5 / 1,3-44,8$ & $8,8 / 1,5-40,0$ & $9,1 / 1,7-31,3$ & $6,9 / 0,9-32,3$ \\
\hline Amonio & $\mu \mathrm{g} / 1$ & $97,5 / \mathrm{ND}-260,0$ & 171,1/ND-540,0 & $111,1 / \mathrm{ND}-320,0$ & $137,0 / \mathrm{ND}-360,0$ \\
\hline Fósforo soluble (como P) & $\mu \mathrm{g} / 1$ & $37,2 / 8,0-93,9$ & $30,3 / 4,6-90,8$ & $30,6 / 6,9-82,0$ & $35,7 / 5,5-80,3$ \\
\hline Cloruro & $\mathrm{mg} / \mathrm{l}$ & $2,3 / 1,1-4,9$ & $2,3 / 1,1-9,0$ & $2,2 / 1,1-6,2$ & $2,3 / 1,1-5,6$ \\
\hline Sulfato $\left(\right.$ como $\left.\mathrm{SO}_{4}\right)$ & $\mathrm{mg} / 1$ & $1,6 / 0,9-4,1$ & $1,7 / 0,9-6,8$ & $1,7 / 0,8-3,1$ & $1,9 / 0,9-3,5$ \\
\hline Sílice reactiva & $\mathrm{mg} / \mathrm{l}$ & $15,4 / 11,5-17,2$ & $15,1 / 8,4-17,1$ & $15,1 / 7,2-17,4$ & $14,9 / 4,0-17,5$ \\
\hline Hierro & $\mathrm{mg} / \mathrm{l}$ & $1,4 / 0,4-4,5$ & $1,4 / 0,4-4,5$ & $1,4 / 0,6-3,9$ & $1,4 / 0,5-3,5$ \\
\hline Potasio & $\mathrm{mg} / 1$ & $1,7 / 1,1-2,8$ & $1,7 / 1,1-2,8$ & $1,7 / 0,7-4,2$ & $1,9 / 1,2-3,6$ \\
\hline Sodio & $\mathrm{mg} / \mathrm{l}$ & $3,3 / 1,7-5,9$ & $3,3 / 1,7-5,7$ & $3,3 / 1,9-5,7$ & $3,8 / 2,0-6,0$ \\
\hline Microcistina & $\mu \mathrm{g} / 1$ & $0,41 /<0,03-0,79$ & $7,17 / 0,16-14,1$ & $0,46 / 0,45-0,46$ & $0,45 / 0,24-0,66$ \\
\hline
\end{tabular}

Tabla 3. Valores promedio, mínimo y máximo de los parámetros fisicoquímicos estudiados durante los estudios de línea de base.

El oxígeno disuelto fluctuó entre 7 y $10 \mathrm{mg} / 1$ y el fósforo soluble varió entre 4,6 $\mu \mathrm{g} / \mathrm{l}$ (Puente, julio de 2006) y 116,0 $\mu \mathrm{g} / 1$ (Puente, setiembre de 2007) (Gráfico 1). Los resultados de las medidas de sustancias orgánicas en agua (compuestos orgánicos adsorbibles: AOX, compuestos clorofenólicos, ácidos resínicos, dioxinas y furanos) se presentan en la Tabla 4.

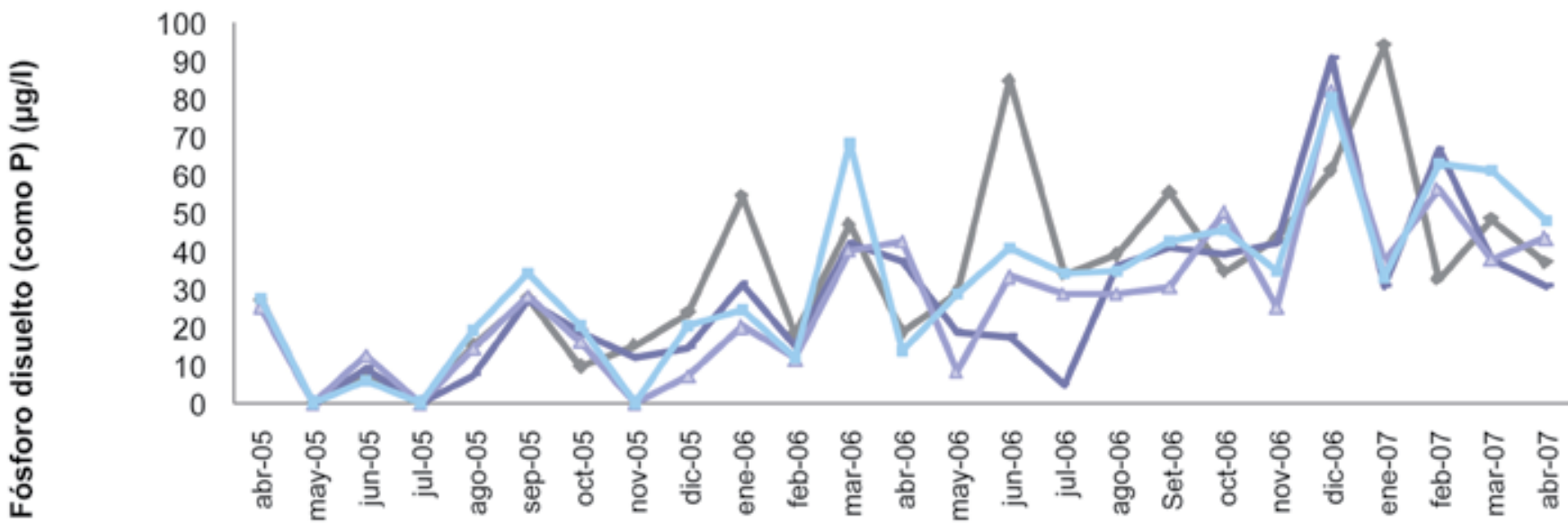

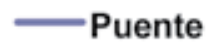

- Fray Bentos

\section{Las Cañas}

Gráfico 1. Concentración de fósforo soluble del agua en los muestreos realizados. 


\begin{tabular}{|l|c|c|c|c|c|}
\hline \multicolumn{1}{|c|}{ Parámetro } & $\begin{array}{c}\text { AOX } \\
\mu \mathrm{g} / 1\end{array}$ & $\begin{array}{c}\text { CI-P } \\
\mu \mathrm{g} / 1\end{array}$ & $\begin{array}{c}\text { RA } \\
\mu \mathrm{g} / 1\end{array}$ & $\begin{array}{c}\text { Fitosteroles } \\
\mu \mathrm{g} / 1\end{array}$ & $\begin{array}{c}\text { PCDD/PCDF } \\
\text { WHO-TEQ pg/1 }\end{array}$ \\
\hline Nuevo Berlín & $8-11$ & $0,089-0,104$ & $27-224$ & $\mathrm{ND}-22$ & $0,00-0,60$ \\
\hline Fray Bentos & $6-12$ & $0,080-0,114$ & $6-183$ & $\mathrm{ND}-2$ & $0,00-0,89$ \\
\hline Las Cañas & $<5-12$ & $0,089-0,185$ & $3-202$ & $\mathrm{ND}-8$ & $0,00-0,96$ \\
\hline
\end{tabular}

Tabla 4. Concentración de AOX, clorofenoles (Cl-P), ácidos resínicos (RA), fitosteroles y dioxinas y furanos (PCDD/PCDF) en agua del Río Uruguay en el período de línea de base.

Durante el período de estudio la comunidad de fitoplancton estuvo representada por 110 taxa, distribuidos en seis clases: Cyanophyceae, Dinophyceae, Euglenophyceae, Chlorophyceae, Bacillariophyceae y Cryptophyceae. El grupo dominante durante la mayor parte del período de estudio fueron Cryptophyceae (fitoflagelados nanoplanctónicos $<30 \mu \mathrm{m}$ ), pero en verano dominaron las cianobacterias. La densidad de fitoplancton presentó un valor mínimo de 32 cél/ml (julio de 2006, en Nuevo Berlín) y un máximo de 4.451 cél/ml (enero de 2007, en Fray Bentos) (Gráfico 2).

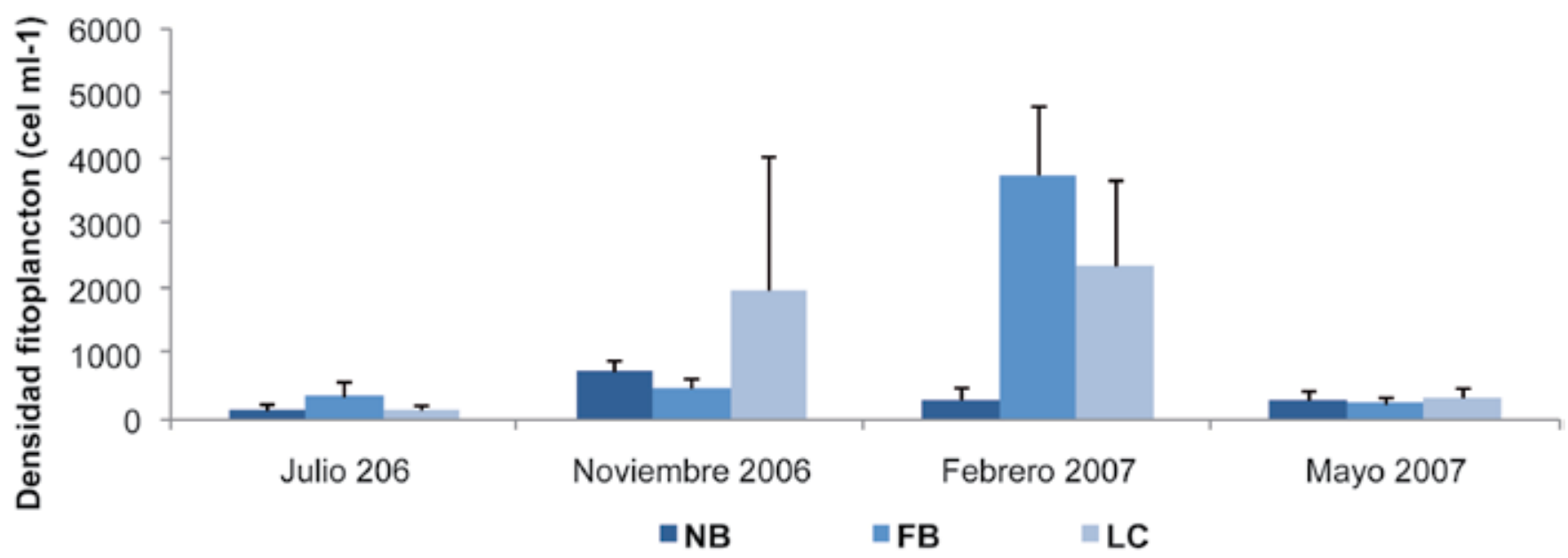

Gráfico 2. Densidad de fitoplancton (cel ml-1) en NB (Nuevo Berlín), FB (Fray Bentos) y LC (Las Cañas) durante el período de estudio.

En la comunidad de zooplancton se encontraron 58 taxa, distribuidos en 36 de Rotíferos, 11 de Cladóceros, 7 de Copépodos y 4 taxa de otros grupos meroplanctónicos, principalmente larvas del molusco Limnoperna fortunei. La comunidad zooplanctónica presentó variación estacional con valores mínimos de densidad en julio de 2007 (0,16 org/l en Nuevo Berlín) y máximos en mayo de 2007 (67,5 org/l en Las Cañas) (Gráfico 3). Esta comunidad presentó una variabilidad estacional definida por dominancia de organismos de menor tamaño (Rotíferos y nauplios de Copépodos) en otoño e invierno, aumento de organismos mayores en primavera (Cladóceros y larvas de Limnoperna fortunei) y codominancia de los diferentes grupos en verano.

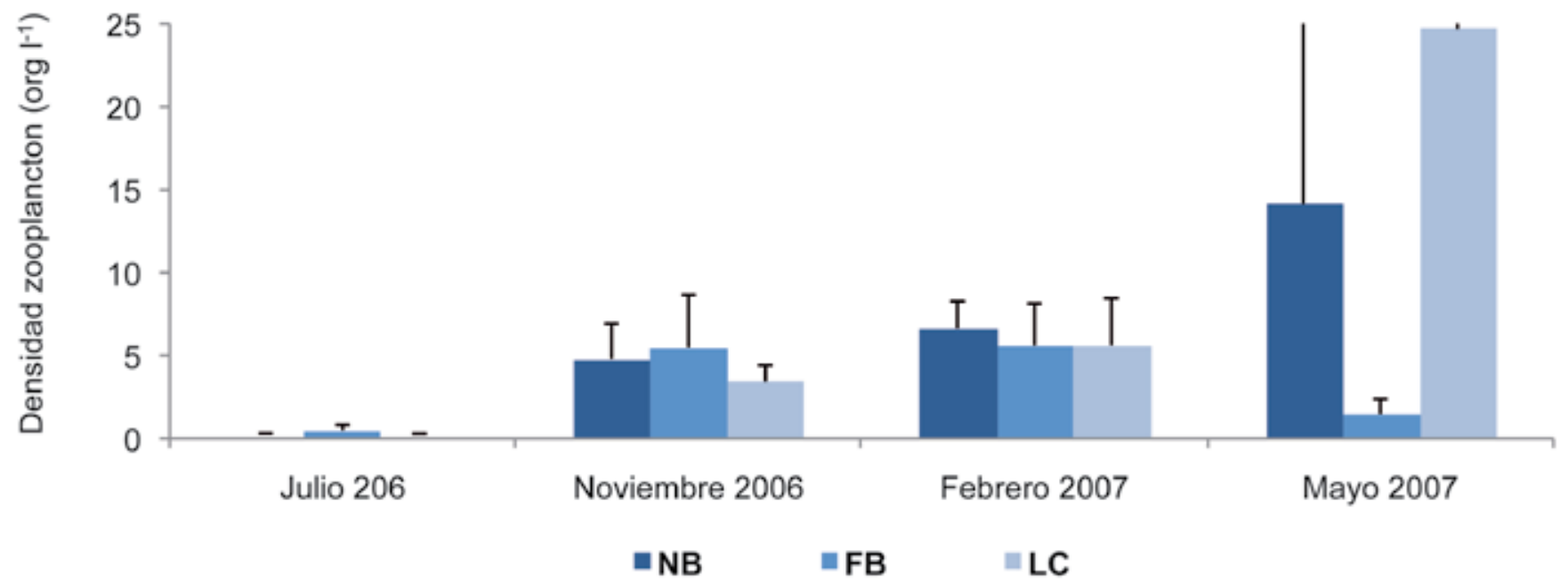

Gráfico 3. Densidad de zooplancton (org I') en NB (Nuevo Berlín), FB (Fray Bentos) y LC (Las Cañas) durante el período de estudio. 
La estructura granulométrica de los sedimentos en la zona de estudio varió entre arena gruesa y limo, predominando las fracciones de arena media, fina y muy fina. Los valores promedio de materia orgánica en sedimentos fueron mayores en octubre $(2,87 \%)$, mientras que mayo fue el mes que presentó menores valores $(0,59 \%)$. Durante el período de estudio se identificaron un total de 18 familias de macroinvertebrados bentónicos. Las más frecuentes pertenecieron a las clases Insecta, con un total de nueve familias (Chironomidae, Ceratopogonidae, Emphidae, Leptoceridae, Caenidae, Polymitrarcidae, Ephemeridae, Elmidae y Gomphidae) y a los moluscos, representados por seis familias. En el período indicado, la densidad de organismos varió entre $9.252 \mathrm{ind} / \mathrm{m}^{2}$ en abril de 2007 y $30.677 \mathrm{ind} / \mathrm{m}^{2}$ en octubre de 2006. La familia Mytilidae, representada por la especie Limnoperna fortunei, fue la que registró las mayores abundancias (Gráfico 4).

En la Tabla 5 se presentan los resultados de los análisis no paramétricos de varianza de las comunidades planctónicas y bentónica. Se analizó la diferencia entre los sitios de muestreo (Nuevo Berlín, Fray Bentos y Las Cañas), las zonas (litoral, central y canal) y entre muestreos.

\begin{tabular}{|ll|c|c|}
\hline \multirow{2}{*}{ Diferencias } & RGlobal & P(\%) \\
\hline \multirow{3}{*}{ Fitoplancton } & Entre sitios (NB, FB, LC) & $-0,108$ & 0,9 \\
\cline { 2 - 4 } & Entre zonas (litoral, central, canal) & $-0,100$ & 0,9 \\
\cline { 2 - 4 } Entre muestreos & 0,926 & 0,1 \\
\hline \multirow{2}{*}{ Zooplancton } & Entre sitios (NB, FB, LC) & $-0,006$ & 0,5 \\
\cline { 2 - 4 } & Entre zonas (litoral, central, canal) & $-0,016$ & 0,6 \\
\cline { 2 - 4 } & Entre muestreos & 0,667 & 0,1 \\
\hline \multirow{2}{*}{ Zooplancton } & Entre sitios (NB, FB, LC) & 0,032 & 2,3 \\
\cline { 2 - 4 } & Entre zonas (litoral, central, canal) & 0,038 & 0,8 \\
\cline { 2 - 4 } & Entre muestreos & 0,052 & 0,3 \\
\hline
\end{tabular}

Tabla 5. Resultados de los análisis no paramétricos de varianza (ANOSIM) de las comunidades planctónicas y bentónica. (NB: Nuevo Berlín, FB: Fray Bentos, LC: Las Cañas).

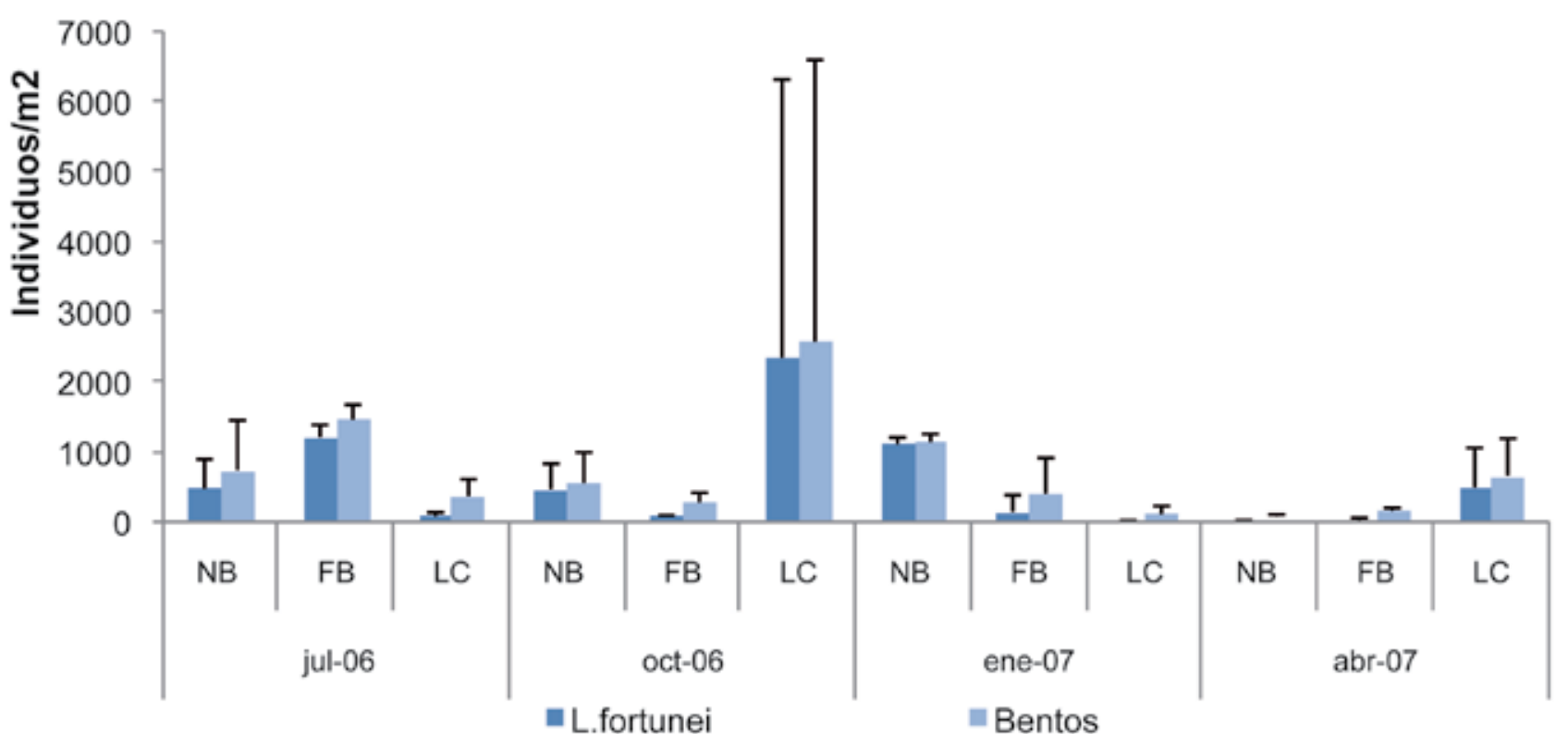

Gráfico 4. Densidad de macrozoobentos y de la especie Limnoperna fortunei (ind $\mathrm{m}^{-2}$ ) en NB (Nuevo Berlín), FB (Fray Bentos) y LC (Las Cañas) durante el período de estudio.

El número de especies capturadas en este estudio en las tres áreas asciende a 56 (Tabla 6) y el número de especies se muestra en el Gráfico 5. Las especies más comunes capturadas en este estudio fueron el Bagre trompudo (Iheringichthys labrosus), Corvina de río (Pachyurus bonariensis), Bagre amarillo (Pimelodus aff. maculatus), Bagarito (Parapimelodus valenciensis), Vieja (Paraloricaria vetula), Vieja (Loricariichthys melanocheilius), Virolito (Apareiodon affinis), Anchoa de río (Lycengraulis grossidens) y varias especies de mojarras (Characidae). Todas ellas han estado presentes en los cinco muestreos realizados.

Excepto en el muestreo de mayo de 2006, el sitio con menor número de especies fue Las Cañas, con una variación de entre 16 y 27. En el área Nuevo Berlín el número de especies varió entre 13-32 y en la bahía Yaguareté (zona de la planta), entre 26 y 30 (Gráfico 5). La media de las capturas, individuos/red y kg/red, es presentada en los Gráficos 6 y 7; se destacan el bajo número y biomasa en el área Las Cañas. 


\begin{tabular}{|c|c|c|c|}
\hline Orden & Familia & Especie & Nombre común \\
\hline \multirow[t]{2}{*}{ Clupeiformes } & Engraulidae & Lycengraulis grossidens & Anchoita \\
\hline & Pristigasteridae & Pellona Flavipinnis & Lacha \\
\hline \multirow[t]{26}{*}{ Characiformes } & Parodontidae & Apareiodon affinis & Virolo \\
\hline & \multirow[t]{3}{*}{ Curimatidae } & Cyphocharax spilotus & Sabalito \\
\hline & & Cyphocharax platanus & Sabalito \\
\hline & & Steindachnerina brevipinna & Sabalito \\
\hline & Prochilodontidae & Prochilodus lineatus & Sábalo \\
\hline & \multirow[t]{3}{*}{ Anostomidae } & Schizodon nasutus & Boga \\
\hline & & Leporinus striatus & Trompa colorada \\
\hline & & Leporinus obtusidens & Boga \\
\hline & \multirow[t]{15}{*}{ Characidae } & Astyanax aff. abramis & Mojarra \\
\hline & & Astyanax eigenmanniorum & Mojarra \\
\hline & & Astyanax aff. fasciatus & Mojarra \\
\hline & & Bryconamericus iheringii & Mojarra \\
\hline & & Bryconamericus stramineus & Mojarra \\
\hline & & Oligosarcus jenynsii & Dientudo \\
\hline & & Oligosarcus oligolepis & Dientudo \\
\hline & & Cynopotamus argenteus & Dientudo jorobado \\
\hline & & Galeocharax humeralis & Dientudo jorobado \\
\hline & & Cheirodon interruptus & Mojarra \\
\hline & & Heterocheirodon yatai & Mojarra \\
\hline & & Brycon orbignyanus & Salmón criollo \\
\hline & & Salminus brasiliensis & Dorado \\
\hline & & Serrassalmus maculatus & Piraña \\
\hline & & Pygocentrus nattereri & Piraña \\
\hline & Acestrorhynchidae & Acestrorhynchus pantaneiro & Dientudo paraguayo \\
\hline & Cynodontidae & Rhaphiodon vulpinus & Machete \\
\hline & Erithrynidae & Hoplias aff. malabaricus & Tararira \\
\hline \multirow[t]{20}{*}{ Siluriformes } & \multirow[t]{8}{*}{ Loricariidae } & Hypostomus alatus & Vieja de agua \\
\hline & & Hypostomus commersoni & Vieja de agua \\
\hline & & Hypostomus laplatae & Vieja de agua \\
\hline & & Pseudohemiodon devincenzii & Vieja de agua \\
\hline & & Ricola macrops & Vieja de agua \\
\hline & & Paraloricaria vetula & Vieja de látigo \\
\hline & & Loricariichthys edentatus & Brujita \\
\hline & & Loricariichthys melanocheilus & Vieja de agua \\
\hline & Aspredinidae & Bunocephalus sp. & Guitarrero \\
\hline & \multirow[t]{2}{*}{ Heptapteridae } & Rhamdia quelen & Bagre negro \\
\hline & & Pimelodella gracilis & Burrito \\
\hline & \multirow[t]{4}{*}{ Pimelodidae } & Iheringichthys labrosus & Bagre trompudo \\
\hline & & Pimelodus aff. maculatus & Bagre pintado \\
\hline & & Pimelodus albicans & Bagre blanco \\
\hline & & Luciopimelodus pati & Patí \\
\hline & \multirow[t]{4}{*}{ Auchenipteridae } & Trachelyopterus teaguei & Torito \\
\hline & & Auchenipterus nuchalis & Buzo \\
\hline & & Ageneiosus militaris & Manduvi \\
\hline & & Ageneiosus inermis & Manduva \\
\hline & Doraridae & Rhinodoras dorbignyi & Marieta \\
\hline \multirow[t]{2}{*}{ Gymnotiformes } & Sternopygidae & Eigenmannia virescens & Banderita \\
\hline & Gymnotidae & Gymnotus sp. & Señorita \\
\hline \multirow[t]{2}{*}{ Atheriniformes } & \multirow[t]{2}{*}{ Atherinopsidae } & Odontesthes perugiae & Pejerrey \\
\hline & & Odontesthes humensis & Pejerrey \\
\hline \multirow[t]{4}{*}{ Perciformes } & \multirow[t]{3}{*}{ Cichlidae } & Crenicichla minuano & Cabeza amarga \\
\hline & & Crenicichla missioneira & Cabeza amarga \\
\hline & & Crenicichla vittatta & Cabeza amarga \\
\hline & Sciaenidae & Pachyurus bonariensis & Corvina de río \\
\hline Pleuronectiformes & Achiridae & Catathyridium jenynsii & Lenguado \\
\hline
\end{tabular}

Tabla 6. Listado de especies de peces (nombre científico y común) encontradas durante el período de estudio. 


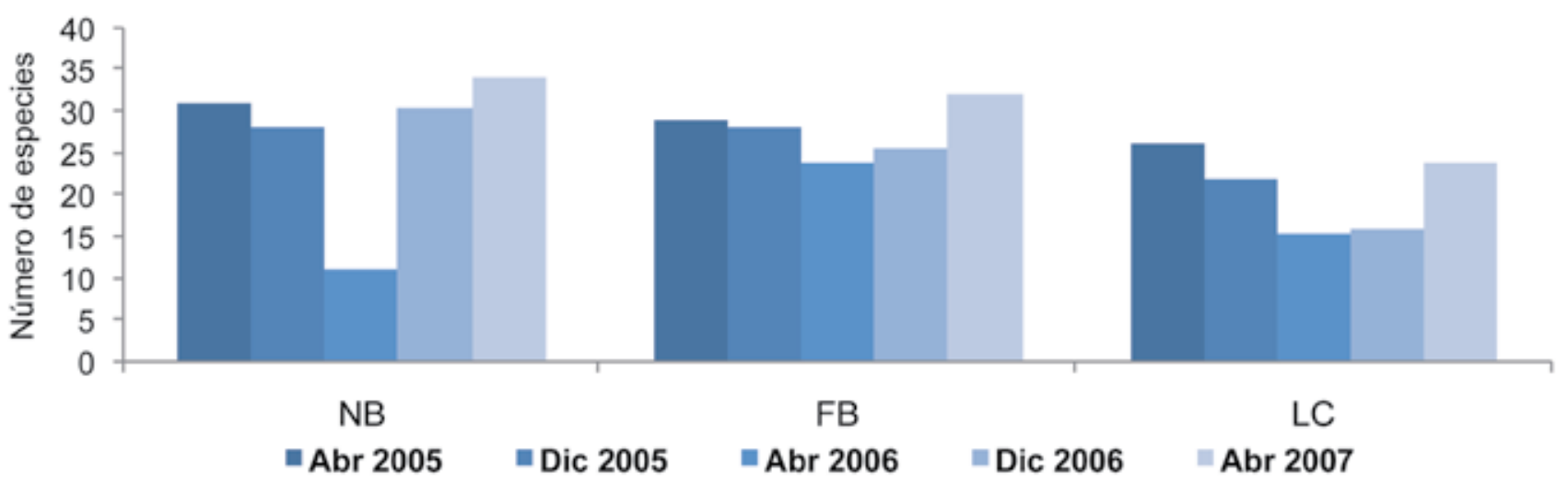

Gráfico 5. Número de especies de peces capturadas en NB (Nuevo Berlín), FB (Fray Bentos) y LC (Las Cañas) durante el período de estudio.

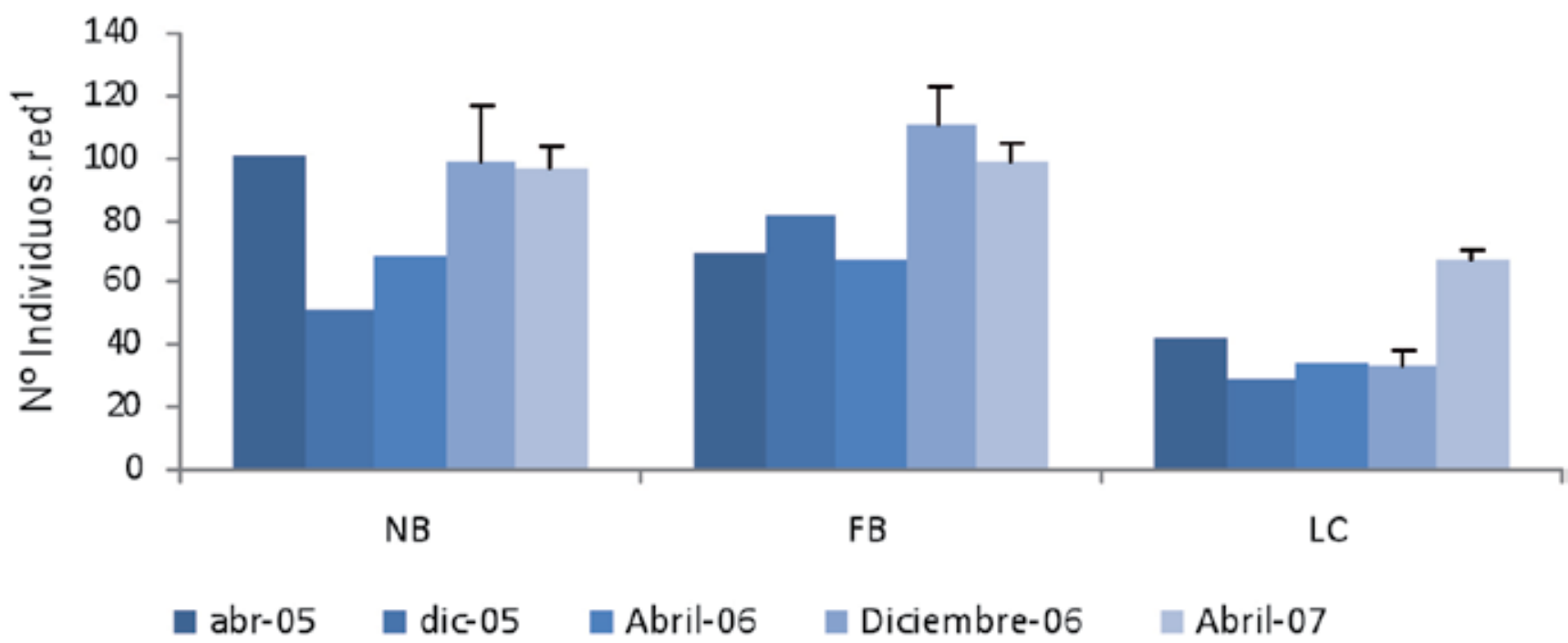

Gráfico 6. Comparación de las capturas promedio (individuos/red) en NB (Nuevo Berlín), FB (Fray Bentos) y LC (Las Cañas) durante el período de estudio.

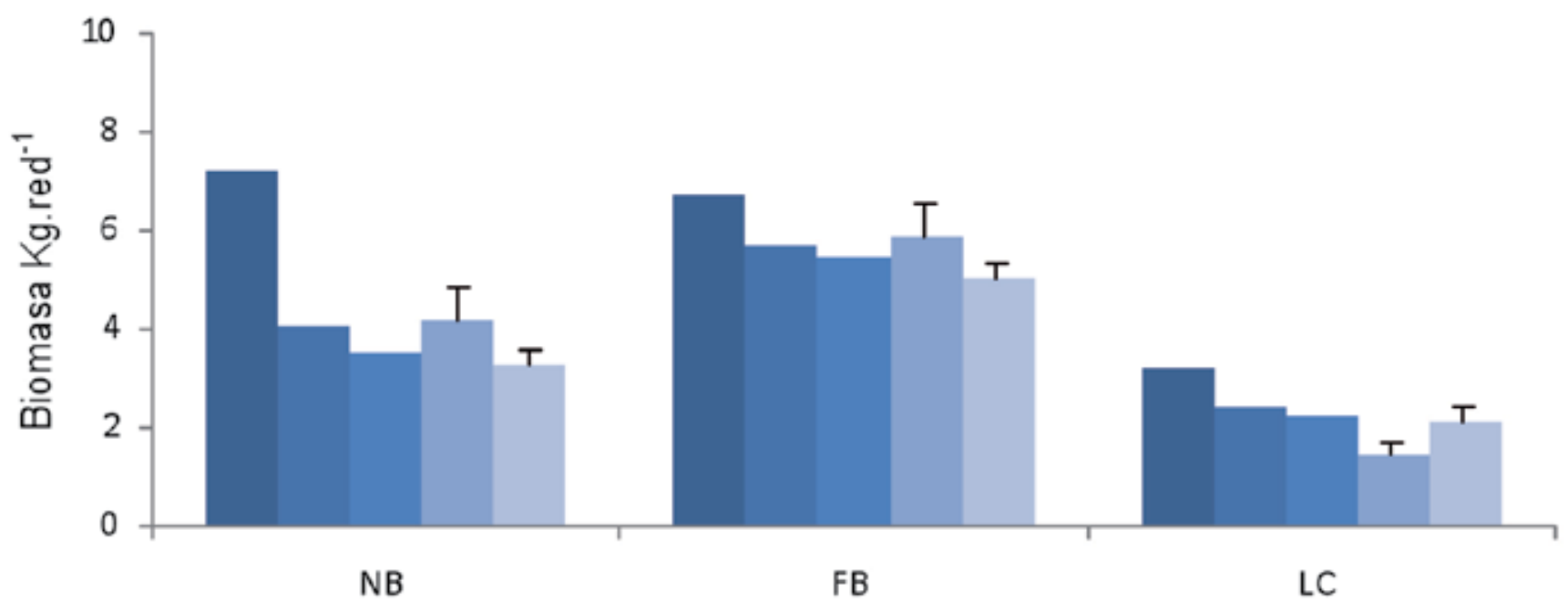

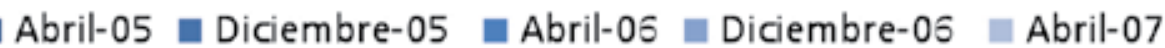

Gráfico 7. Comparación de unidades de captura (kg/red) en NB (Nuevo Berlín), FB (Fray Bentos) y LC (Las Cañas) durante el período de estudio. 
En función de la selección del Bagre trompudo como especie indicadora se estudiaron índice somático, factor de condición e índice gonadosomático en las tres zonas, en abril de 2007 (Tabla 7).

\begin{tabular}{|l|c|c|c|}
\hline & Nuevo Berlín & Fray Bentos & Las Cañas \\
\hline CF & $1,54 \pm 0,10$ & $1,44 \pm 0,14$ & $1,30 \pm 0,11$ \\
\hline LSI & $0,66 \pm 0,16$ & $0,67 \pm 0,11$ & $0,63 \pm 0,12$ \\
\hline GSI & $0,51 \pm 0,25$ & $0,52 \pm 0,21$ & $0,53 \pm 0,15$ \\
\hline
\end{tabular}

\begin{tabular}{|l|c|c|c|c|}
\hline & $\begin{array}{c}\text { Fenoles } \\
\text { clorados (bilis) } \\
\text { ng/g bilis d.w. }\end{array}$ & $\begin{array}{c}\text { Ácidos } \\
\text { resínicos (bilis) } \\
\mu \mathrm{g} / \mathrm{g} \text { bilis d.w. }\end{array}$ & $\begin{array}{c}\text { Fitoesteroles } \\
\text { (bilis) } \\
\mu \mathrm{g} / \mathrm{g} \text { bilis d.w. }\end{array}$ & $\begin{array}{c}\text { PCDD/PCDF } \\
\text { (músculo) } \\
\text { WHO-TEQ pg/g f.w. }\end{array}$ \\
\hline Nuevo Berlín & $70-1560$ & $2-1800$ & $330-1170$ & $0,11-0,30$ \\
\hline Fray Bentos & $80-1480$ & $8-1080$ & $12-1440$ & $0,08-1,01$ \\
\hline Las Cañas & $160-1190$ & $4-660$ & $130-1640$ & $0,19-0,49$ \\
\hline
\end{tabular}

\section{Discusión}

El oxígeno disuelto se encontró por encima del mínimo recomendado por la normativa nacional $(5 \mathrm{mg} / \mathrm{l})$ y el fósforo total se presentó consistentemente por encima de los valores límite guía propuestos por la normativa nacional.

Las concentraciones medias de los compuestos orgánicos adsorbibles (AOX) fue de $9 \mu \mathrm{g} / \mathrm{l}$, salvo en una ocasión (setiembre de 2005), cuando se observaron valores puntuales superiores a $200 \mu \mathrm{g} / \mathrm{l}$ en la zona del Puente y en la de Fray Bentos. Las concentraciones de fitosteroles, tales como sitosterol, campesterol y sitostanol, se mantuvieron por debajo del límite de cuantificación $(50 \mu \mathrm{g} / \mathrm{l})$.

Las concentraciones de AOX, compuestos clorofenólicos, ácidos resínicos y dioxinas pueden ser consideradas bajas y básicamente en los mismos niveles basales naturales medidos en cursos de aguas en Escandinavia (Tana, 2010).

Las dioxinas y furanos se mantuvieron por debajo del nivel guía de $10 \mathrm{pg} / \mathrm{l}$ (Health Canada, 2010). El OCDD es el congener que predomina en el ambiente (Green et al., 2004, Rappolder et al., 2005) y el de menor toxicidad entre los PCDD, con un factor de equivalencia tóxica (TEF) de la Organización Mundial de la Salud de 0,0003 respecto al 2,3,7,8-TCDD (Van den Berg et al., 2006). En áreas agrícolas y forestadas, como las que predominan en esta región, la deposición atmosférica es la fuente más probable de PCDD/Fs en sedimentos (Wentz et al., 1998) y, por lo tanto, también en agua. El OCDD es el congener predominante en las emisiones de camiones diesel, de vehículos a nafta sin plomo y de la combustión de madera (Cleverly et al., 1997).

Los metales pesados tóxicos (arsénico, cadmio, cinc, cobre, cromo, mercurio, níquel y plomo) se encontraron durante todo el monitoreo por debajo de los máximos establecidos por la normativa nacional para aguas Clase 1 (destinadas a abastecimiento público).

La riqueza de taxa de fitoplancton fue menor a la encontrada en estudios anteriores a lo largo del Río Uruguay y la abundancia fue similar a la encontrada en estudios anteriores en el mismo río (O'Farrell e Izaguirre, 1994; CELA, 2006). La comunidad de fitoplancton presentó variación estacional, típica de los ríos de esta región, con valores máximos en verano en todos los puntos de muestreo. Durante el período estival CELA registró la presencia de una floración de Microcystis aeruginosa en la zona de estudio (2006). Los valores de nutrientes (fósforo soluble y compuestos nitrogenados, Tabla 3), las altas temperaturas $\left(2{ }^{\circ} \mathrm{C}\right.$ promedio en enero) y las condiciones de bajo caudal (valores menores a $1000 \mathrm{~m}^{3} \mathrm{~s}^{-1}$ durante todo el período estival) fueron factores que favorecieron el crecimiento de cianobacterias. En enero se detectaron en todos los puntos densidades que estuvieron en el orden de las $20.000-30.000 \mathrm{cé} 1 / \mathrm{ml}$ (CELA, 2006), en tanto la microcistina-LR detectada en agua alcanzó en ese mes los $0.79 \mu \mathrm{g} / \mathrm{L}$
Tabla 7. Valores promedio de Factor de Condición (CF), Índice Somático (LSI) e Índice Gonadosomático (GSI) de hembras de Bagre trompudo de tamaño de clases 15-22, capturados en las tres áreas de estudio.

Tabla 8. Variación de las concentraciones de fenoles clorados, ácidos resínicos y fitoesteroles analizados en bilis y PCDD/PCDF en músculo de diferentes especies de peces analizados durante el período de estudio.

en Nuevo Berlín. La espuma de color verde se visualizó y permaneció en superficie durante el período estival (Figura 2), y alcanzó en febrero valores de microcistina superiores de $14.1 \mu \mathrm{g} / \mathrm{l}$ en Fray Bentos.

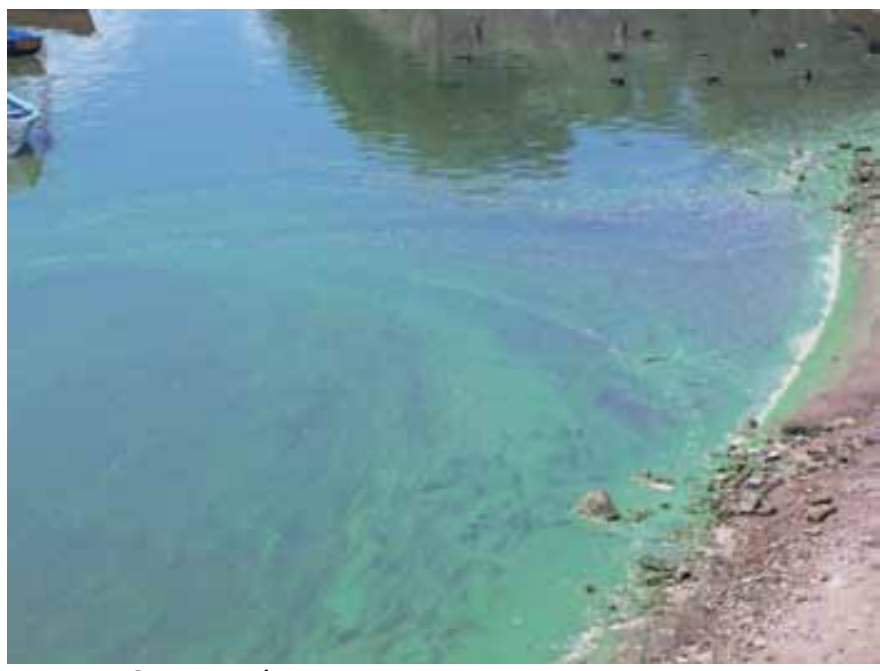

Figura 2. Observación costera de mancha verde producida por acumulación de cianobacterias durante el período estival del año 2006.

El aumento de cianobacterias en verano, principalmente de la especie Microcystis aeruginosa, no sólo marca signos de eutrofización sino que también implica riesgos para la salud por su capacidad de generar hepatotoxinas (Chorus y Bartram, 1999). La dominancia de los fitoflagelados nanoplanctónicos el resto del año se debe a que son organismos pequeños, con alta relación superficie/volumen y altas tasas de reproducción, adaptados a condiciones de alta concentración de material suspendido y disminución de la transparencia del agua (Klaveness, 1988). La riqueza de taxa de zooplancton fue menor a la encontrada previamente en los ríos Uruguay, Paraná y Paraguay (Paggi y José de Paggi, 1990; Chalar et al., 1993, 2002; Frutos et al., 2006) y se observó un patrón similar que en dichos ríos: predominio de especies de Rotíferos y baja presencia de Cladóceros. La dominancia de Rotíferos se debe a su característica de tener generación de vida corta y alta tasa de reproducción, lo que reduce los impactos de pérdida por advección (Pace et al., 1992).

La abundancia de zooplancton estuvo dentro del rango presentado en el estudio realizado el año anterior por CELA (2006) en las mismas zonas de estudio. No obstante, el máximo registrado en este trabajo fue menor. Las mayores densidades se produjeron por la alta presencia de larvas de Limnoperna fortunei, especie de molusco cuyos primeros estadios de vida forman parte de la comunidad de zooplancton. Es una 
especie exótica, originaria de China e introducida en la región en los años 90 mediante el agua de lastre de las embarcaciones (Darrigran y Pastorino, 1995). En Uruguay esta especie fue hallada por primera vez en 1994 en costas del Río de la Plata (Scarabino y Green, 1995) y a los pocos años ya se encontraba en cuatro de las seis principales cuencas de nuestro país (Brugnoli et al., 2005).

Los principales factores reguladores de las comunidades planctónicas son físicos (intensidad de la luz), químicos (concentración de nutrientes), biológicos (predación y competencia) e hidrológicos (caudales y tiempo de residencia) (Reynolds, 2006; Moss et al., 1989). En sistemas lóticos los factores más importantes son los hidrológicos, principalmente el caudal y sus efectos, como la turbulencia y el tiempo de residencia (Reynolds, 2006; Pace et al., 1992). Las comunidades planctónicas no presentaron diferencias significativas a escala espacial entre zonas de estudio ni puntos de las transectas, a diferencia de la escala temporal, en la que las diferencias se atribuyeron a las variaciones de caudales entre muestreos. En el caso del fitoplancton la temperatura jugó un rol importante como modulador de la abundancia de la comunidad.

Las concentraciones de materia orgánica en sedimentos fueron similares a las relevadas en esta zona durante el período 2005 - 2006 por CELA. Los valores de densidades de organismos bentónicos encontrados en este estudio se encuentran dentro de los rangos relevados por Ezcurra de Drago et al. (2004) en la zona baja del Río Paraguay. $\mathrm{Al}$ igual que la comunidad planctónica, la de bentos tampoco presentó diferencias significativas espaciales entre sitios ni zonas de muestreo. A pesar de que no se observaron cambios temporales significativos en la comunidad de macroinvertebrados, las abundancias fueron mayores en octubre y julio. Según LATU (2010), el caudal del Río Uruguay sería el principal factor regulador de los cambios espaciales y temporales observados en la abundancia de la fauna macrobentónica y la matriz sedimentaria.

En el tramo inferior del Río Uruguay, Devincenzi y Teague (1942) mencionan la presencia de aproximadamente 86 especies de peces. Además, Delfino et al. (1997) citan cinco especies más y Charbonier et al. (2002), 34 más. El total de especies ronda las 125, con la probable presencia de dos especies introducidas: carpa (Cypriniformes, Cyprinus carpio) y esturión (Acipenseriformes, Acipenser baeri, no confirmada realmente). Esta es la zona más productiva del Río Uruguay en términos de biomasa (Spinetti, 1999). Las especies de mayor importancia comercial son el sábalo (Prochilodus lineatus), la boga (Leporinus obtusidens), el patí (Luciopimelodus pati) y el dorado (Salminus brasiliensis). En base a los resultados se observó una importante variación en la aparición de las especies en las tres zonas.

En términos generales, la comunidad de peces presentó variación en la aparición de las especies en las tres zonas a lo largo del periodo de muestreo. Dentro de esta comunidad, P. valencienesis presenta una elevada variabilidad en sus abundancias, pudiendo afectar la representación de las diferentes clases de tallas. Por otra parte, estos elevados cambios en las abundancias entre muestreos pueden estar indicando migraciones locales o de mayor extensión.

Los estudios de base de la comunidad de peces indican que la mejor especie para ser seleccionada como indicadora, debido a sus elevadas abundancias en los diferentes periodos de estudio en los tres sitios, es el Bagre trompudo (Iheringichthys labrosus). A pesar de la amplia distribución geográfica, existen muy pocos trabajos que traten sobre la especie propuesta para ser evaluada como indicadora (I. labrosus). En la represa de Itaipú, estado de Paraná, Brasil, los estudios indican que es una especie no migratoria, que no presenta cuidado parental y se reproduce tanto en el embalse como en sus tributarios (Santos et al., 2004).

El contenido en bilis de peces de compuestos clorofenólicos y resinas ácidas, entre otras, han sido ampliamente utilizados como medidas para establecer los niveles de exposición a efluentes de plantas de celulosa, por ejemplo en Finlandia y Suecia (Grahn et al., 1991; Tana et al., 1994; Johnsen et al., 1995; Tana, 2004). Las principales razones para el análisis de estos compuestos en bilis son la toxicidad para los peces y las concentraciones que pueden llegar a ser del orden de $10^{5}$ con respecto a las encontradas en el agua (proceso de bioconcentración). Su presencia también indica que estos compuestos han sido procesados por el sistema de detoxificación de los peces (Oikari y Holmbom, 1986). Las concentraciones de compuestos clorofenólicos encontradas consisten principalmente en clorofenoles y pueden ser consideradas bajas. Dentro de este grupo los más abundantes fueron 2,4-diclorofenol, 2,4,6-triclorofenol y 2,3,6-triclorofenol, los cuales se ha demostrado que son ubicuos en aguas ricas en humus y formados por la acción de microorganismos (Grimvall et al., 1994).

$\mathrm{Al}$ analizar el factor de condición e índice gonadosomático no se detectaron diferencias estadísticamente significativas entre las tres zonas $(\mathrm{KW}, \mathrm{p}>0.05)$ y lamentablemente no existen trabajos previos en esta especie para el Río Uruguay que permitan realizar comparaciones con los datos obtenidos. Las concentraciones de ácidos resínicos pueden ser consideradas como niveles basales naturales si tomamos como referencia los cursos de agua escandinavos. Las elevadas concentraciones observadas en noviembre de 2006 en el área Las Cañas corresponden al ácido abiético en una muestra proveniente de un pool de cinco bagres trompudos (I. labrosus). Datos similares fueron observados en abril de 2007.

Los resultados de las concentraciones de los compuestos clorofenólicos, resinas ácidas y fitoesteroles analizadas en la bilis de diferentes especies capturadas en los tres sitios mostraron una gran variación entre especies, en el espacio y en el tiempo. Concentraciones excepcionalmente elevadas de cloroguayacoles y clorocatecoles fueron detectadas en Nuevo Berlín en diciembre de 2005 en una muestra compuesta de tres Sábalos (P. lineatus, especie migratoria y de importancia en la pesca artesanal).

El consejo de la Unión Europea ha establecido en el Reglamento 199/2006 unos niveles máximos de determinados contaminantes en los productos alimenticios. El nivel máximo de PCDD/PCDF en pescado y productos pesqueros se fijó en 4,0 pg WHO-PCDD/F-TEQ/g peso fresco. Los valores máximos de $\mathrm{PCBs}$ se establecen en dicho reglamento combinados con $\mathrm{PCDD} / \mathrm{PCDF}$ en un límite admisible de 8,0 pg WHO-PCDD/F-PCB- TEQ/g peso fresco. Hasta el momento los valores encontrados en Uruguay para dioxinas y PCB no superan estos límites. La ingesta diaria total recomendada por la Unión Europea es de 1-4 pg/kg de peso corporal, y esto incluye un margen de seguridad en un factor de 10. Basado en esta recomendación no habría ningún tipo de limitación al consumo humano de los peces estudiados en cuanto a las concentraciones de dioxinas y furanos. Sin embargo, se debe prestar especial atención debido a estudios recientes que muestran elevadas concentraciones de endosulfan en varias de las especies aquí estudiadas (Zaldúa et al., 2010). Todos los datos de concentraciones de dioxinas observados se encuentran muy por debajo de los valores recomendados, y son hasta el momento los primeros datos disponibles para las concentraciones de dioxinas en los peces del Río Uruguay.

\section{Conclusiones}

Existen pocos antecedentes en la zona de estudio, por lo que fue fundamental realizar una línea de base completa, con diversos parámetros abióticos y bióticos en conjunto, para luego poder evaluar si existieron cambios en el ambiente a partir de la puesta en funcionamiento de la planta de celulosa. La calidad físicoquímica del agua fue uniforme en las zonas analizadas y los parámetros estudiados presentaron valores similares en todas las zonas de estudio. Las concentraciones estuvieron dentro de los valores límite guía de la normativa nacional, con la excepción del fósforo, el cual superó los máximos sugeridos. El área de estudio mostró señales de eutrofización debido a las altas concentraciones de fósforo en la columna de agua y a la presencia en altas concentraciones de especies de cianobacterias (principalmente Microcystis aeruginosa) en la comunidad de fitoplancton.

La comunidad de plancton estuvo dominada por grupos adaptados a la turbidez y turbulencia: fitoflagelados nanoplanctónicas (fitoplancton) y rotíferos (zooplancton). Los valores máximos de densidad de zooplancton y bentos se observaron por la alta presencia del molus- 
co exótico invasor de origen asiático Limnoperna fortunei, la especie bentónica dominante en el período de estudio.

Las comunidades de plancton y bentos presentaron variabilidad temporal entre los muestreos y no se observó un patrón espacial que diferencie las zonas de estudio.

La comunidad de peces fue similar en las tres zonas de estudio y la mayor cantidad de especies fue obtenida en la bahía de Yaguareté, cercana a la zona de Fray Bentos y a la futura planta de celulosa. Las menores capturas fueron obtenidas en la zona de Las Cañas, aguas debajo de la planta a la altura de la desembocadura del arroyo Caracoles chico. Los peces capturados se encontraban en buen estado y las investigaciones de la bilis de peces indicaron que las concentraciones de compuestos clorofenólicos, ácidos resínicos y fitoesteroles se encontraban en niveles en los que no era de esperar efectos agudos o crónicos sobre esta comunidad.

Las concentraciones de dioxinas y furanos en músculo de peces se encontraron por debajo de los niveles recomendados por normativa internacional y el grupo más tóxico de las dioxinas (TCDD), por debajo de los límites de detección. En base a las concentraciones observadas y las recomendaciones internacionales no habría limitaciones al consumo humano de los peces estudiados para los contaminantes analizados.

La ausencia de diferencias significativas entre las tres zonas estudiadas en la etapa de línea de base es relevante para la posterior evaluación de la presencia de impactos de la planta de celulosa.

\section{Reconocimientos}

Agradecemos especialmente a la empresa UPM S A. que nos permitió utilizar los datos generados en el marco de los servicios prestados, a las personas que junto a nosotros participaron en los muestreos, principalmente Marcelo Bado, Pablo Morales y Gonzalo Useta, y a todos los compañeros del LATU que de alguna forma contribuyeron con este trabajo. Agradecemos a Diego Larrea, Nicolás Vidal, Carlos Iglesias, Iván Gonzales-Bergonzoni y Roberto Ballabio por su participación en el muestreo y procesamiento de peces. También queremos agradecer a los dos revisores anónimos que permitieron mejorar este trabajo.

\section{Referencias}

- ALI, M.; SREEKRISHNAN, T.R. Aquatic toxicity from pulp and paper mill effluents: a review. En: Advances in Environmental Research. 2001, 5:175-196.

- BRUGNOLI, E.; CLEMENTE, J.; BOCCARDI, L.; BORTHAGARAY, A.; SCARABINO, F. Update and prediction of golden mussel (Limnoperna fortunei): distribution in the principal hydrographic basin of Uruguay. En: Anais da Academia Brasileira de Ciencias. 2005, 77(2):1-10.

- BURT, R. Soil survey laboratory methods manual. v. 4.0. Lincoln: NRCS, 2004.

- CARU. COMISIÓN ADMINISTRADORA DEL RÍO URUGUAY. Cuenca del Río Uruguay [En línea]. Paysandú: CARU, s.d. [Consulta marzo 2009]. Disponible en: www.caru.org.uy

\section{- CELA. CENTROS DE ESTUDIOS LIMNOLÓGICOS}

APLICADOS. Establecimiento de una línea de base de las comunidades de fitoplancton, zooplancton y bentos en el Río Uruguay (desde Nuevo Berlín a Las Cañas), depto. Río NegroUruguay. s.1.: CELA, 2006.

- CHALAR, G.; DE LEÓN, L.; DE LEÓN, R.; FABIÁN, D.; GORGA, J. Evaluación de la eutrofización del Embalse de Salto Grande. Análisis de las relaciones entre los parámetros físico-químicos y biológicos. Informe final de la primera etapa. Montevideo: CTM-SG; Universidad de la República, 1993.
- CHALAR, G.; DE LEÓN, L.; PARADISO, M.; BRUGNOLI, E.; CLEMENTE, J. Dinámica de la eutrofización del embalse Salto Grande: informe del periodo setiembre de 2000-marzo 2002. Montevideo: Facultad de Ciencias, 2002.

- CHARBONIER, E.; D'ANATRO, A.; GARCÍA, D.; LOUREIRO, M.; TEIXEIRA DE MELLO, F. Medio Biótico: peces. En: CAYSSIALS, R.; MANEYRO R.; PERES-MILES, F. Pautas para la elaboración de un plan de manejo para el área de Esteros de Farrapos. Primera Parte: medio fisico y fauna. Montevideo: DINAMA; Facultad de Ciencias, 2002.

- CHORUS, I.; BARTRAM, J. Toxic Cyanobacteria in water. A guide to their public health consequences, monitoring and management. London: WHO; E\&FN Spon, 1999.

- CLEVERLY. D.; SCHAUM. J.; SCHWEER, G.; BECKER, J.; WINTERS, D. The Congener Profiles of Anthropogenic Sources of Chlorinated Dibenzo-P-Dioxins and Chlorinated Dibenzofurans in the U.S. Bayreuth: Ecoinforma Press, 1997.

- DARRIGRAN, G.; PASTORINO, G. The recent introduction of asiatic bivalve, Limnoperna fortunei (Mytilidae) into South America. En: Veliger. 1995, (38):183-187.

- DAVENPORT, J.; SAYER, MD. Physiological determinant of distribution in fish. En: Journal of Fish Biology. 1993, 43(Supplement A):121-145.

- DELFINO, R.; AMESTOY, F.; SVERLIJ, S.; SPINETTI, M.; ESPINACH, ROS A; FOTI, R.; CHEDIAK, G.; BELLAGAMBA, M. Estructura de las comunidades de peces. Conservación de la fauna íctica en el embalse de Salto Grande. Montevideo: Comisión Administradora del Río Uruguay; Comisión Técnica Mixta de Salto Grande, 1997.

- DEVINCENZI, G.; TEAGUE, G. Ictiofauna del Río Uruguay medio. En: Anales del Museo de Historia Natural de Montevideo. 1942, $2^{\mathrm{a}}$ Serie 5(4).

- EZCURRA DE DRAGO, I.; MARCHESE, M.; WANTZEN, K.M. Spatial patterns in Benthic invertebrate community structure along the Lower Paraguay Floodplain System. En: Arch. Hydrobiol. 2004, 160:347-374.

- FRUTOS, S.; POI DE NEIFF, A.; NEIFF, J. Zooplankton of the Paraguay River: a comparison between sections and hydrological phases. En: Ann. Limnol. Int. J. Lim. 2006, 42(4):277-288.

- GRAHN, O.; TANA, J.; MONFELT, C.; HÄRDIG, J.; LEHTINEN, K. Environmental impact of two Swedish bleached kraft pulp mills as determined by field surveys. En: Proceedings of the 1st Symposium on Environmental Fate and Effects of Bleached Pulp Mill Effluents. Stockholm: s.n. 1991. pp. 248-256.

- GREEN, N.; HASSANIN, A.; JOHNSTON, A.; JONES, K. Observations on historical, contemporary, and natural PCDD/Fs observations on historical, contemporary, and natural PCDD/Fs. En: Environmental Science and Technology. 2004, 38(3):715-723.

- GRIMVALL, A.; LANIEWSKI, K.; BOREN, H.; JOHANSSON, S.; KAUGARE, S. Organohalogens of natural or unknown origin in surface water and precipitation. En: Toxicology Environmental Chemistry. 1994, 46(3):183-196.

- HEALTH CANADA. Current canadian objectives, guidelines and regulations [En línea]. Ottawa: Health Canadá, [s.d.]. [Consulta noviembre 2010]. Disponible en: http://www.hc-sc.gc.ca/ewh-semt/ pubs/contaminants/psl1-lsp1/dioxins_furans_dioxines_furannes/ guidelines-canada-objectifs-eng.php.

- HERING, D.; MOOG, O.; SANDIN, L.; VERDONSCHOT, P. Overview and application of the AQEM assessment system. En:

Hidrobiología. 2004, 516(1-3):1-20.

- HUTSON, D.; ROBERTS, T. Environmental fate of pesticides. v. 7. 
s.1.: John Wiley \& Sons, 1994. (Progress in Pesticide Biochemistry and Toxicology)

- JOHNSEN, K.; MATTSSON, K.; TANA, J.; STUTHRIDGE, T.R.; HEMMING, J.; LEHTINEN, K. Uptake and elimination of resin acids and physiological responses in rainbow trout exposed to total mill effluent from integrated newsprint mill. En: Environmental Toxicology and Chemistry. 1995, 14(9):1561-1568.

- KARR, J.R. Assessment of biotic integrity using fish communities. En: Fisheries. 1981, 6:21-27.

- KARRASCH, B., PARRA O., CID H., MEHRENS M., PACHECO P., URRUTIA R., VALDOVINOS C., ZAROR C. Effects of pulp and paper mill effluents on the microplankton and microbial selfpurification capabilities of the Biobío River, Chile. En: Science of the Total Environment. 2006, 359:194-208.

- KLAVENESS, D. Ecology of the Cryptomonadida: a first review. En: SANDGREN, C.D. Growth and reproductive strategies of freshwater phytoplankton. Cambridge: Cambridge University Press, 1988.

- LARSSON, D.G.J; FÖRLIN, L. Male-biased sex ratios of fish embryos near a pulp mill: temporary recovery after a short-term shutdown. En: Environmental Health Perspectives. 2002, 110(8):739742.

- LATU. Estudio de las comunidades de fitoplancton, zooplancton y macrozoobentos en el tramo inferior del Río Uruguay (Nuevo Berlín, Fray Bentos y Las Cañas). Montevideo: LATU, 2007. (Informe de asesoramiento; 952512)

- LATU. Tercer año de estudio de las comunidades biológicas $y$ variables abióticas en el tramo inferior del Río Uruguay. Montevideo: LATU, 2010. (Informe de asesoramiento; 1135493)

- LAWTON, L.; EDWARDS, CH.; CODD, G. Extraction and highperformance liquid chromatographic method for the determination of microcystins in raw and treated waters. En: Analyst. 1994, 119(7):1525-1530.

- MOSS, B.; BOOKER, I.; BALLS, H.; MANSON, K. Phytoplankton distribution in a temperate floodplain lake and river system. Hydrology, nutrient sources and phytoplankton biomass. En: Journal of Plankton Resources. 1989, 11:813-838.

- O'FARRELL, I.; IZAGUIRRE, I. Phytoplankton ecology and limnology of a River Uruguay Lower Basin (Argentina). En: Arch. Hydrobiol. Suppl. 1994, 99:(1/2):155-179.

- OIKARI, A.; HOLMBOM, B. Assessment of water contamination by chlorophenolics and resin acids with the aid of fish bile metabolites. En: POSTON, T.M.; PURDY, R. Aquatic toxicology and environmental faste. v. 9. Philadelphia: ASTM, 1986. pp. 225-267. (ASTM STP 921)

- PACE, M.; STUART, E.; LINTS, D. Zooplankton in advective environments. The Hudson River community and a comparative analysis. En: Can. J. Fish. Aquat. Sci. 1992, 49:1060-1069.

- PAGGI, J.C.; JOSE DE PAGGI, S.B. Zooplankton of the lotic and lentic environments of the middle Paraná River. En: Acta Limnol. Brazil. 1990, 3:685-719.

- RAPPOLDER, M.; BRÜDERS, N.; SCHRÖTER- KERMANI, C. Comparison of congener patterns and TEQs in environmental and human samples. En: Organohalogen Compounds. 2005, 67:20862089.

- REYNOLDS, C.S. The ecology of phytoplankton. Cambridge: Cambridge University Press, 2006. (Ecology, Biodiversity and Conservation).

- SANTOS, J.; BAZZOLI, N.; RIZZO, E.; SANTOS, G. Reproduction of the catfish Iheringicthys labrosus (Lütken) (Pisces, Siluriformes) in Furnas reservoir, Minas Gerais, Brazil. En: Revista Brasileira de Zoología. 2004, 21(2):139-200.
- SCARABINO, F.; VERDE, M. Limnoperna fortunei (Dunker 1857) en la costa uruguaya del Río de la Plata (Bivalvia; Mytilidae). En: Comunicaciones Malacológicas del Uruguay. 1995, 7(66-67):374375.

- SPINETTI, M. Evaluación de los recursos pesqueros mediante técnica hidroacústicas y pesca exploratoria. En: COMISIÓN ADMINISTRADORA DEL RÍO URUGUAY. Primeras Jornadas sobre Conservación de la Fauna Íctica en el Río Uruguay, (Paysandú 22-23 de noviembre de 1999). Paysandú: C.A.R.U, 1999. pp. 9-12.

- TANA, J. Aquatic environmental impact evaluation of the April Riau Industrial Complex, Indonesia. Espoo: CTS, 2004. (AF-CTS report April 2004).

- TANA, J. Concentrations of resin acids, chlorinated phenols and plant sterols in fish from Rio Uruguay. Monitoring studies in the recipient of Botnia pulp mill. [s.1.]: [s.n.], 2010. (ÅF-Consult report ENVI-569, June 2010).

- TANA, J.; ROSEMARIN, A.; LEHTINEN, K.; HÄRDIG, J.; GRAHN, O.; LANDNER, L. Assessing impacts on Baltic coastal ecosystem with mesocosm and fish biomarker tests. A comparison of new and old wood pulp bleaching technologies. En: Science of the Total Environment. 1994, 145(3):213-234.

- TEIXEIRA-DE MELLO, F. Monitoreo de la pesca artesanal en el Río Uruguay a través de información generada por pescadores de la zona de Nuevo Berlín, Fray Bentos y Las Cañas (periodo 2007-2009). En: Informe técnico presentado a la empresa Botnia, 2010.

- UNITED STATES ENVIRONMENTAL PROTECTION AGENCY. United States of America. Environmental Monitoring and assessment Program (EMAP). Laboratory methods manual: estuaries. Washington: USEPA, 1995. (EPA/620/R-95/008).

- UNITED STATES ENVIRONMENTAL PROTECTION AGENCY. Summary of Biological Assessment Programs and Biocriteria Development for States, Tribes, Territories, and Interstate Commissions: Streams and Wadeable Rivers. Washington: USEPA, 2002. (EPA-822-R-02-048).

- UTERMHÖL. Zur Vervollkommnung der quantitativen Phytoplankton Methodik. Mitteilungen der internationalen Vereinigung für theoretische und angewandte En: Limnologie. 1958, 9:1-38.

- VAN DEN BERG, L. ; SCHILDER, M.; DE VRIES, H.; LEEGWATER, P.; VAN OOST, B. Phenotyping of aggressive behaviour in Golden Retriever dogs with a questionnaire. En: Behav Genet. 2006, 36(6):882-902.

- ZALDÚA, N.; RÍOS, M.; TEIXEIRA-DE MELLO, F.; GONZÁLEZBERGONZONI, I.; MIGUELE, C.; ROSANO, A.; SPINELLI, W. Evaluación participativa de niveles de plaguicidas en peces de interés comercial del sitio RAMSAR. En: RÍOS, M.; ZALDÚA, N.; CUPEIRO, S. Evaluación participativa de plaguicidas eb el sitio Ramsar, Parque Nacional Esteros de Farrapos e Islas del Rio Uruguay. Montevideo: Vida Silvestre, 2010. pp. 59-76.

- WENTZ, D.A.; BONN, B.A.; CARPENTER, K.D.; HINKLE, S.R.; JANET, M,L.; RINELLA, F.A.; UHRICH, M.A.; WAITE, I.R.; LAENEN, A.; BENCALA, K.E. Water quality in the Willamette Basin, Oregon, 1991-95. Denver: U.S. Department of the Interior, 1998. (U.S. Geological Survey Circular; 1161). 